\title{
Relationship between type 2 diabetes mellitus and coronary artery lesion characteristics: a single center study
}

\author{
Laxman Dubey*, Sogunuru Guruprasad, Gangapatnam Subramanyam \\ Department of Cardiology \\ College of Medical Sciences and Teaching Hospital, Bharatpur, Chitwan, Nepal
}

\section{Citation \\ Dubey L, Guruprasad S, Subramanyam G. Relationship between type 2 diabetes mellitus and coronary artery lesion characteristics: a single center study. Nepalese Heart Journal $2013 ; 10(1): 20-22$.}

\section{Keywords}

\section{Coronary artery disease; multivessel disease,} type Clesion; type 2 diabetes mellitus

\begin{abstract}
Background: Coronary atherosclerotic burden is excessive in diabetic patients. The aim of present study was to assess the relationship between diabetes mellitus and plaque characteristics in patients with coronary artery disease.

Methods: The coronary angiograms of 223 patients with significant obstructive lesions during April 2011 to May 2013 in College of Medical Sciences and Teaching Hospital were analyzed. The coronary lesions of patients with and without type 2 diabetes were compared.
\end{abstract}

Results: 81 diabetic and 142 non-diabetic patients had significant coronary artery disease. Diabetic patients had more multivessel (64.2\% versus $55.6 \%, p<0.05)$, and small vessel disease (74.1\% versus $54.9 \%, p<0.05)$ than nondiabetic patients. Moreover, complex Type C lesion was significantly higher in the diabetic patients as compared to the nondiabetic patients (55.5\% versus $29.6 \%, p<0.01$ )

Conclusions: Complex coronary lesions were significantly more common in diabetic patients than in nondiabetic patients

\section{INTRODUCTION}

Cardiovascular disease is the leading cause of mortality worldwide, and approximately half of all cardiovascular deaths are attributed specifically to coronary artery disease (CAD). Extensive epidemiologic research has led to identification of cardiovascular risk factors such as high low density lipoprotein and low high density lipoprotein levels, type 2 diabetes, systemic hypertension, cigarette smoking and obesity. ${ }^{1,2}$ Diabetes affects more than 150 million adults worldwide, and prevalence is expected to reach 300 million by $2025 .{ }^{3}$ Type 2 diabetes is an established and powerful predictor of CAD that has evolved into a major public health concern in recent years. ${ }^{4}$

It has been shown that diabetic patients have more plaques, which appeared to have characteristics of plaque vulnerability and a different composition of plaques than nondiabetic patients for those presenting with acute coronary syndrome (ACS). ${ }^{5}$ Diabetic patients have more triple-vessel and less single-vessel CAD than nondiabetics. ${ }^{6}$ The aim of this study was to assess the plaque characteristics in

\footnotetext{
*Corresponding Author:

Laxman Dubey

Department of Cardiology

College of Medical Sciences and Teaching Hospital,

Bharatpur, Chitwan, Nepal

E-mail: dubeylax@yahoo.com
} 
culprit lesions in diabetic patients with CAD.

\section{METHODS}

We retrospectively analyzed 223 consecutive patients, ranging from 27 years to 91 years old, who underwent coronary angiography (CAG) from April 2011 to May 2013 in our center. CAG was done both from femoral and radial arteries access site. Written informed consent was obtained in all cases. An occlusion of $\geq 70 \%$ was considered as significant obstructive lesion (significant stenosis). If there was $\geq 70 \%$ stenosis in a single vessel, it was considered single vessel disease (SVD), if in two vessels it was considered double vessel disease (DVD), and if there was significant stenosis involving all three vessels then it was termed triple vessel disease (TVD). Both DVD and TVD were collectively termed as multivessel disease. Angiographic coronary artery stenosis was assessed by visual means only. Vessel stenosis of $<70 \%$ was considered non-critical CAD. Patients with normal coronaries as well as noncritical CAD were excluded from the study. Patients were classified into those with type 2 diabetes and without diabetes. Diabetes was defined as a fasting blood glucose $>126 \mathrm{mg} / \mathrm{dl}$ and/or on treatment. Coronary artery lesions were characterized into Type A, Type B, and Type C according to the ACC and AHA derived lesion classification ${ }^{7}$ with special focus on Type C lesions. Data collection with statistical analysis was done using statistical package for social sciences version 13 for windows.

\section{RESULTS}

A total of 452 patients had undergone CAG during two years period. Age range from 27 years to 91 years. Among them 180 were normal coronaries or non-critical lesions and excluded from the study. Remaining 272 had significant obstructive disease, however; we could obtain the data of 223 patients only and were subsequently analyzed in the present study. Among those 223 patients who were included in the study, $81(36.3 \%)$ were diabetic and rest 142 $(63.7 \%)$ were nondiabetic.

There was no significant difference in the baseline data between diabetic and nondiabetic patients. The incidence of multivessel, smaller-vessel disease in diabetic patients was higher than that in nondiabetic patients (Table 1). Moreover, Type C lesion was significantly higher in the diabetic patients as compared to the nondiabetic patients $(55.5 \%$ versus $29.6 \%, \mathrm{p}<0.01$ ) (Table 1).

\section{DISCUSSION}

Type 2 diabetes has been associated with an increased risk of cardiovascular disease. Moreover, individuals with diabetes manifest symptoms more severely and earlier in life compared to those without diabetes. The present study focused on angiographic characteristics of coronary atherosclerosis, with particular attention to the prevalence of complex Type $\mathrm{C}$ lesions in patients who underwent $\mathrm{CAG}$ because of CAD. We found that the prevalence of multivessel and small vessel lesion was significantly higher in patients with type 2 diabetes than in those without diabetes. Importantly, complex Type $\mathrm{C}$ lesion was significantly higher in type 2 diabetes patients than in nondiabetes patients.

Previous studies have reported that the triplevessel disease is more frequent in patients with type 2 diabetes, ${ }^{8-10}$ although other studies have demonstrated that the angiographic profiles are similar among diabetic and nondiabetic patients. ${ }^{11,12}$ Diabetic patients have a higher risk of complications and a worse prognosis than nondiabetic subjects during coronary interventions. ${ }^{13}$ The higher prevalence of multivessel and complex Type $\mathrm{C}$ lesions noted in the present study could be a factor contributing to this less favorable outcome. The more severe and extensive coronary artery lesions, and poorer collateral circulation in diabetic patients reported in another study ${ }^{14}$ may have contributed to the less favorable clinical outcomes of this patient population.

The mechanism of the increased severity of CAD in type 2 diabetics than in nondiabetics in the study is unclear. Insulin resistance in patients with type 2 diabetes often leads to hyperinsulinemia, which in turn causes a variety of other abnormalities, including elevated triglyceride levels, low levels

Table 1. Characteristics of patients with coronary artery disease with diabetes mellitus (DM) and without DM (non-DM)

\begin{tabular}{llll}
\hline & DM $(\mathrm{n}=81)$ & Non-DM $(142)$ & $\mathrm{P}$ \\
\hline Age, years (mean \pm SD) & $55 \pm 6$ & $58 \pm 7$ & NS \\
Female sex, $n(\%)$ & $21(25.9 \%)$ & $34(23.9 \%)$ & NS \\
Multivessel disease, $n(\%)$ & $52(64.2 \%)$ & $79(55.6 \%)$ & $<0.05$ \\
Type A and B lesion, $n(\%)$ & $36(44.4 \%)$ & $100(70.4 \%)$ & $<0.01$ \\
Type C lesion, $n(\%)$ & $45(55.5 \%)$ & $42(29.6 \%)$ & $<0.01$ \\
Small vessel disease, $n(\%)$ & $60(74.1 \%)$ & $78(54.9 \%)$ & $<0.05$ \\
\hline
\end{tabular}


of high density lipoprotein cholesterol, enhanced secretion of very low density lipoprotein, disorders of coagulation, increased vascular resistance and hypertension. ${ }^{15,16}$ All of these consequences contribute to the development of atherosclerosis and CAD.

\section{CONCLUSION}

Type 2 diabetes has a significant adverse effect on the anatomy of coronary arteries, causing more multivessel and extensive coronary artery disease than in nondiabetic patients. The higher prevalence of complex coronary lesions, such as multivessel and complex Type $\mathrm{C}$ lesions, may help to explain the poor prognosis of CAD in diabetic patients.

\section{REFERENCES}

1. Wilson PW, D'Agostino RB, Levy D, et al. Prediction of coronary heart disease using risk factor categories. Circulation 1998;97:1837-47.

2. Yusuf S, Hawken S, Ounpuu S, et al. Effect of potentially modifiable risk factors associated with myocardial infarction in 52 countries (the INTERHEART study): case-control study. Lancet 2004;364:937-52.

3. Mokdad AH, Ford ES, Bowman BA, et al. Prevalence of obesity, diabetes, and obesity-related health risk factors, 2001. JAMA 2003;289:76-9.

4. Bonow RO, Gheorghiade M. The diabetes epidemic: a national and global crisis. Am J Med 2004;116:2S-10S.

5. Hong YJ, Jeong MH, Choi YH, et al. Plaque characteristics in culprit lesions and inflammatory status in diabetic acute coronary syndrome patients. JACC Cardiovasc Imaging. 2009;2:339-49

6. Dortimer AC, Shenoy PN, Shiroff RA, et al. Diffuse coronary artery disease in diabetic patients: Fact or fiction? Circulation 1978;57:133-6.

7. Ryan TJ, Faxon DP, Gunnar RM, et al. Guidelines for percutaneous transluminal coronary angioplasty. A report of the American College of Cardiology/American Heart Association Task Force on Assessment of Diagnostic and Therapeutic Cardiovascular Procedures (Subcommittee on Percutaneous Transluminal Coronary Angioplasty). Circulation 1988;78:486-502

8. Ozerkan F, Ceyhan C, Baris N, et al. Angiographic comparison of the severity and distribution of coronary artery disease in patients with and without diabetes mellitus. Turk J Endocrinol Metab 2001;2:71-4.

9. Uddin SN, Malik F, Bari MA, et al. Angiographic severity and extent of coronary artery disease in patients with type 2 diabetes mellitus. Mymensingh Med J 2005;14:32-7.

10. Vigorito C, Betocchi S, Bonzani G, et al. Severity of coronary artery disease in patients with diabetes mellitus. Angiographic study of 34 diabetic and 120 nondiabetic patients. Am Heart J 1980;100:782-7.

11. Hochman JS, Phillips WJ, Ruggieri D, et al. The distribution of atherosclerotic lesions in the coronary arterial tree: Relation to cardiac risk factors. Am Heart J 1988;116:1217-22.

12. Pajunen $\mathrm{P}$, Nieminen $\mathrm{MS}$, Taskinen $\mathrm{MR}$, et al. Quantitative comparison of angiographic characteristics of coronary artery disease in patients with noninsulin-dependent diabetes mellitus compared with matched nondiabetic control subjects. Am J Cardiol 1997;80:550-6.

13. Baris N, Akdeniz B, Uyar S, et al. Are complex coronary lesions more frequent in patients with diabetes mellitus? Can J Cardiol 2006; 22: 935-7.

14. Abaci A, Oguzhan A, Kahraman S, et al. Effect of diabetes mellitus on formation of coronary collateral vessels. Circulation 1999; 99: 2239-42.

15. Saltiel AR. Series introduction: the molecular and physiological basis of insulin resistance: emerging implications for metabolic and cardiovascualr diseases. J Clin Invest 2000;106:163-4.

16. Wu T, Wang L. Angiographic characteristics of the coronary artery in patients with type 2 diabetes. Exp Clin Cardiol 2003;7: 199-200. 